\title{
Application and Research of Big Data in E-Commerce Enterprises. Amazon as the Case
}

\author{
Yujie Chen ${ }^{1} \mathrm{a}^{*}$ \\ ${ }^{1}$ Wuhan University of Technology, China \\ a547255872@qq.com
}

Keywords: Component; Big data; Application; Electronic commerce enterprise; Case analysis

\begin{abstract}
With the change of people's living habit and consumption concept, the demand of highly personalized products and accurate products is increasing. This condition has a huge impact on the enterprise which cannot adapt to the transformations in the Big Data Era. This paper based on the supply chain which analyzes the electric commercial enterprises, Amazon, provides the recommendation about big date applications to other enterprises by using case evidence. We hope that other firms are able to absorb the experience, to promote the development of their enterprises and the economic progression in Big Data Era.
\end{abstract}

\section{Introduction}

In the twenty-first Century, Big data has become one of the most important resources of enterprises and countries. As for the exploration and development of big data, many European countries and Japan are at the forefront of the world. The government of each country has enhanced the development of big data to a strategic height, developed big data industry, established database, data center, etc. IDC, a research company in the United States, has released a report that the global technology and services market of big data will maintain an annual growth of $31.7 \%$ in the next several years. Thus, the total size of 2016 is expected to reach 28 billion 300 million dollars. According to New Moore's Law, the world may have the amount of data 35ZB in 2020.[1]

Amazon.com currently is the world's largest e-business platform. To use Amazon as the research object will help other business enterprises learn experience, develop big data.

\section{The Big Data Process of Amazon}

Amazon's big data process is divided into three stages: artificial collection and application, primary recommendation system, which was more complex to build the data system, such as figure 1.and logistics information system, new data recommendation system and logistics management system, such as figure 2 .

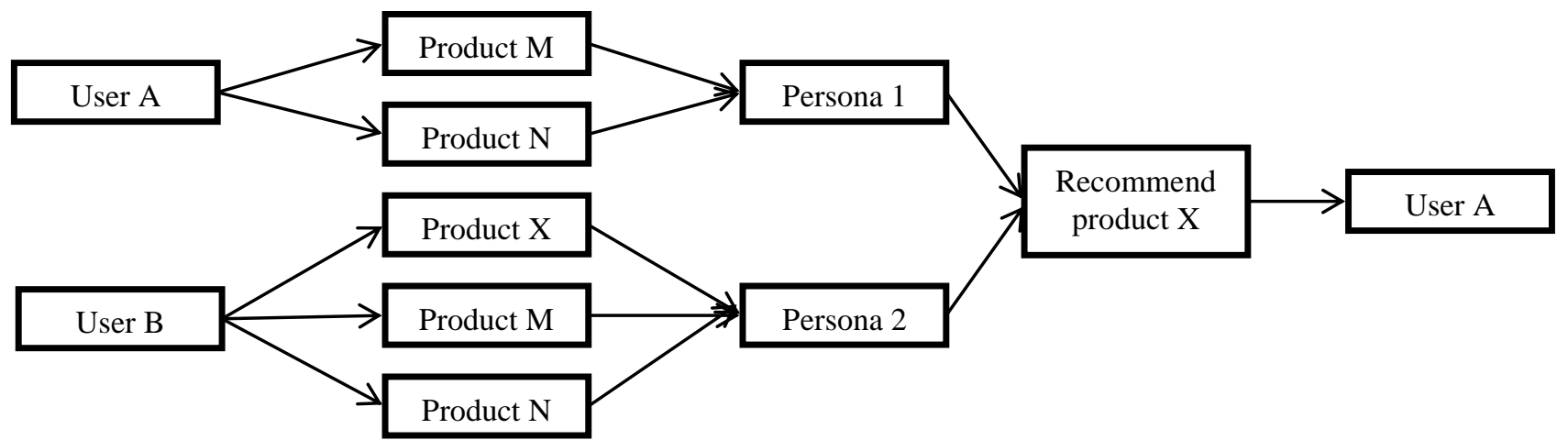

Figure 1. Recommendation system of user association 


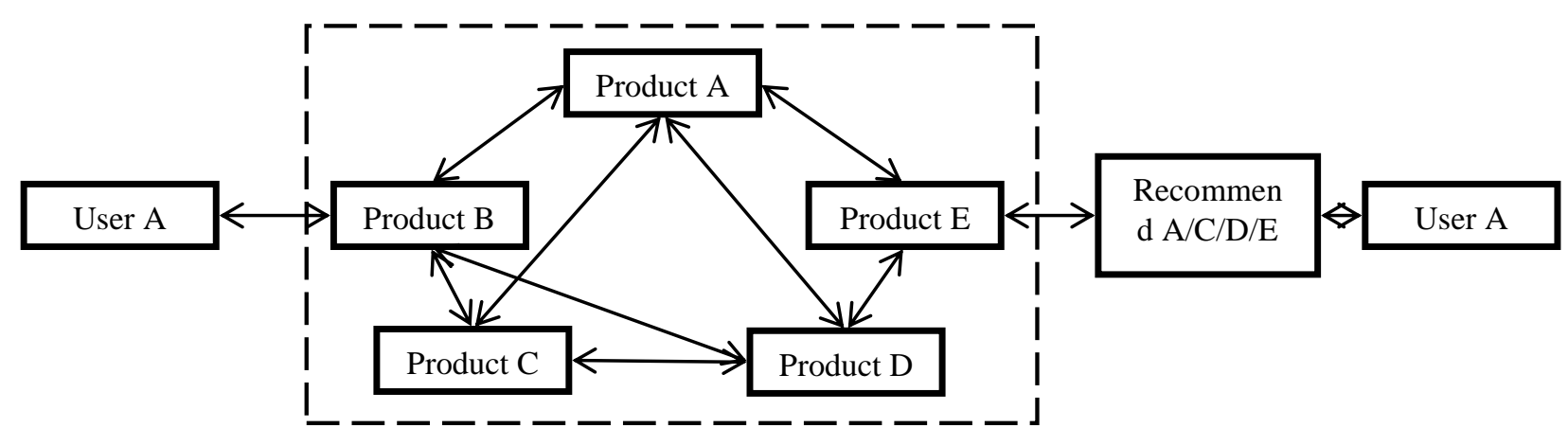

Figure 2. Recommendation system of product association

\section{The Big Data Application of Amazon}

This chapter based on the supply chain analyzes the big data application of Amazon for three parts.

Demand Forecasting and Production Sales. Mining user information to achieve precision marketing

The recommended way for E-Commerce website includes three kinds:

a) According to the user's browsing, searching and other related behavior to recommend.

b) According to the users' shopping cart or list of favorite to recommend.

c) According to the user's historical purchase record to do EDM or membership marketing.

Amazon is not trying to sell things, but to meet the needs of customers, to help customers to buy things, and standing on the consumer's position to help them.

Amazon not only records the search content for each user's product browsing details, staying time, goods comparison, and final purchase; but also pushes some new features or information of free products and questionnaire for customer. They want to understand and analyze customer, to find the user basic information, hobbies, habits and potential demand.

Share Consumer Demand to Achieve a Win-Win Supply Chain. Amazon's supply chain is complete, through many years of accumulated huge data to predict consumer demand, and calculate the order quantity of a product in a certain region for a certain period. It can ensure the inventory of each warehouse, and active reaction order. Then the information will be provided to members of the supply chain.[2]

For the major enterprises in the supply chain, Amazon's demand forecasting can not only help them to certain extent, control inventory, reduce costs, but also improve the company's profits and achieve the entire supply chain members of a win-win situation.[3]

Warehousing and Distribution Analysis. By using big data, random placement to improve efficiency

Unlike most businesses, the goods in Amazon warehouse is randomly placed according to the principle of saving space.[4]

On one hand, the advanced Amazon system will design the optimal display according to good's size. As a result, the Amazon average processing has saved 3 minutes for each order, and the efficiency has increased by at least 3 to 4 times. On another hand, advanced inventory management system can realtime tracking to each order in the center of the operating state of operation. In addition, the core suppliers can see the sales of their goods in Amazon's backstage system and replenish stocks in time and make production plan.

Forecast order quantity to prepare in advance

The "double 11" of two years ago, Amazon had subjected to the logistics problems. Over the past "double 11", amazon purchase from overseas directly, sales was four times bigger than last year. It was 
understood by all orders in Amazon. China had been dispatch and delivery, and the on-time delivery rate was more than $98.4 \%$.

Xue Xiaolin, the CLO of Amazon, said, "Despite the double 11 day surge in orders, thanks to the powerful operation ability of the amazon big data, we can accurately predict the specific category of every hour and orders. Then we have sufficient prepare in advance to ensure efficient order processing and distribution."

Self-run logistics combined with the third party logistics

Amazon's own logistics has the national integration of distribution network and national allocation model, which is based on the big data and the cloud computing. After receiving the order information, the powerful operation system will automatically operation hundreds of possible delivery path, and calculate the fastest delivery time quickly. The Amazon operations center can accurately calculate each commodity cost, delivery time and profit rate to calculate the fastest and lowest cost way.

Data in the Quality, Assurance Service and Evaluation System Analysis. Big data filtering valuable comments

As early as Amazon was founded at the beginning, Bezos established a customer feedback system which allows customers to give negative reviews and retain them. Negative feedback does not necessarily lead to bad effect, it could be a chance to improve. For example, after changing the supplier, the feedback of product becomes worse. This means that the goods quality problems, and enterprise can choose to replace the supplier to meet customer demand.

Amazon's customer feedback mechanism is also a social platform. There are some reviews below to the product. Consumers here can share the experience, analysis of products, mutual exchange. They also can send e-mail to Amazon to consult.

Compared with Taobao, the users can ask the author questions and evaluate the usefulness of this review. After big data filtering, Amazon displays some long comments, which will be more helpful to the user to buy goods. Amazon's evaluation system will be more useful for customers to choose comments.

Allowing customers to choose to comment on whether it is useful, the system will put more useful comments in front of the popular reviews to facilitate customer reference.

Amazon quality supervision for third party sellers' goods.

As online shopping become more convenient, more and more consumers buy goods from Internet. But according to the "lemon theory"[5], in on-line shopping market whose information is really unique, it is easy to appear the phenomenon of bad money drive out good-- low-quality goods to drive out highquality goods.[6]Amazon is a B2C electrical business enterprise which main is self-goods. But there are also some businessmen use the amazon platform to sell their products. Thus, Amazon cannot control the quality of them.

\section{Case Inspiration}

It is important for other E-Commerce enterprises to study application of Big data in Amazon. So this part will analysis the advantages and disadvantages it have.

\section{Advantage}

Leaders Focus on Big Data. One of the main mission of big data is to help business decisions and guides the operation. The rise rate of apply big data depends on whether the leader focus on it.Some people say that Bezos is like a "tyrant", strong, arrogant, self-centered, but his business vision is no doubt. He continued to improve the business model and develop new business which makes shareholders shocked.[7]There is no doubt that the success of Amazon has a close relationship with Bezos. As a technical leader, he was able to grasp the direction of enterprises, to develop big data 
exploration earlier, and he is a real far-sighted leader. he carry out early big data exploration, is undoubtedly a forward-looking leader. The key of leadership is to use personal charm to get their approval, so as to lead the followers to create more value.[8]If there is nothing to copy in Amazon, of course, it's Bezos His idea is amazon's guiding ideology, and his leadership is the key to the success of amazon. Leaders must determine the direction of development of the enterprise to develop the longterm planning of the enterprise, which is the general strategy of the enterprise. [9]For example, the global expansion strategy, investment in China strategy, etc.

Enterprise Culture Develop Big Data. Amazon's guiding ideology is:

- Customers first

In order to give customers the perfect experience, Amazon research data to help customers to buy goods, save time, adjust warehousing, design and calculate logistics lines, faster delivery of goods; ensure consumer groups and help advertisers choose advertising. For instance, when customers have a complaint email, leaders will add a question mark and forward it to related staff. It's like a ticking time bomb. At this time, the team must put down the hands of all things immediately and remove the bomb in a few hours .

- Constant innovation

Amazon has special management that can make every employee participate in innovation. There was a small innovation that in warehouse each receiving station has a button to ask question when they are in trouble. If you press the button, an assistant will help you to solve the problem within 10 seconds. Amazon's culture is to encourage innovation.

The ultimate goal of innovation is to improve the customer experience. Because Bezos is a technical personnel, his understanding of technology is more than the number of management personnel. Thus he has a very positive attitude to innovation and asks his staff to innovate. Compared with most of the ECommerce, the pursuit of Amazon is technological innovation and development. Above all Amazon's core competitiveness is constantly updated technology.

Market Trends Promote the Development of Big Data. Many countries have increased the big data to the height of the national strategy, such as "big data research and development initiative" in the U.S., a "global big data program" in the United Nations and "the data rights campaign" in the British.[10]

The Chinese government attaches great importance to the development of big data. In August 2015, government released a notice that "Action plan for promoting the development of big data". It is not only clear the development of the guiding ideology of big data, the development goals and tasks, but also for the future development and application of big data pointed out the direction. In October 2015, the Central Committee released "the thirteenth five plan" which proposed to implement the national big data strategy, and promote sharing resources of big data.

Cloud Computing Technology Provides Conditions. Cloud computing is a platform for big data, big data is the core of cloud computing. The development of cloud computing create a platform for the application of big data. In July 2002, Amazon AWS (Amazon Web Services) appeared on the market. It could provide the Amazon's hardware and software resources to the third party users. Cloud computing technology, in particular, a variety of storage services (e.g. Data mining) will promote the development of big data.

The rise of cloud computing create the most suitable environment for big data applications. You need a few days and nights to calculate before, but now only need a few seconds to get the results. Cloud computing is the necessary process of the application for big data, is the analysis of large data platform and is an important method of mining data. 
Table 1 main services of Cloud computing

\begin{tabular}{|l|l|}
\hline Product & Describe \\
\hline Storage service (S3) & It can provide data storage services for third parties. \\
\hline Simple DB & Providing a simple storage service for cloud computing applications \\
\hline FPS & Amazon payment system \\
\hline MTURK & Remote control system \\
\hline EC2 & User can rent cloud computer system \\
\hline CDN & It can distribute information quickly to end users \\
\hline
\end{tabular}

\section{Disadvantages}

Problems of Talent Cultivation. Big data is not only a revolution in the field of science and technology, but also involved all over the world's revolution in all industry. In 2014, Accenture Management Consulting Company's survey found that the United States needs a great amount of big data of senior management personnel. The demand of all data analysis of executive positions must be increased rapidly. They believe that in the near future, the demand for data analysis of talent will become increasingly urgent. It is important that an enterprise can keep up with the pace of the era of big data, and with enough data scientist.

Data Sources Problem. Before the Internet appeared, the traditional industries such as banking and telecommunications companies are the largest data owners. But the Internet era, electricity suppliers and social networking become data magnate. Even the free WIFI system has become a new way to get data.

Quality Problem of Data. Big data means the amount of data is large, but it does not mean there are lots of useful data. Experian Data Quality survey shows that $89 \%$ of the data management departments focus on data quality solutions, which lead that most of the companies in this area of technology have invested more than $\$ 500$ thousand.[11]

Data quality needs to be controlled from the source. Each link needs to carry out data validation and data cleaning and screen to ensure the quality of data and reduce the difficulty of data analysis and processing. Data quality management is a comprehensive process, which cannot be simply solved by technical means. In addition, it needs the enterprise's high attention to develop data processing technology. Then it can provide high-quality data for enterprises or improve the data quality.

Data Mining and Analysis Problem. Data analysis is the most important step to show the value for the data. If enterprise has data but never analyze the data, the data will be no use and no value. The ability of data analysis is to obtain valuable information from the data. Not only staff needs to be sensitive to data, but also needs to be familiar with the business enterprise.

Data Security Problem. Big data technology can deal with a wide range of problems, but also will encounter more and more information security troubles. For instance, when a user registered, the electrical business enterprise will collect the user's name, phone number, id number, bank card number, address and other personal information. They can also know the user's shopping habits, social tools, and even users can know the personal information of friends besides.

\section{Suggestions}

Focus on Big Data Technology. "Focus on the development of big data technology" means we need to strengthen the understanding of the importance of large data, and vigorously develop big data research. Not only to change the concept of enterprise high-level development, but also to enhance the 
awareness of big data. So that, when they heard the decision made by the board of directors, there will be fewer contradictions.

Training Data Scientists. If there is no outstanding talent as the framework to support the development of enterprises, the enterprise will be difficult to take off in a industry. The reason why Amazon is success because there are a lot of data scientists, data analysts and other very good people work in it.

In order to achieve success in the application of big data in the E-Commerce industry, they need to do the following points:

Firstly, introducing talents constantly to renovate and improve business operation so as to meet the demands of company further development.

Secondly, attention should be paid to the training of data personnel, and create external environment to meet the growth of talent and development needs. The third one is setting up the performance evaluation mechanism to enhance the competitive awareness of employees. Last but not least, enterprises should create a better working environment for employees to increase the sense of belonging.3) Create enterprise culture

Good corporate culture can not only enhance the enterprise staff's sense of belonging and increase the team spirit, but also relate to the brand value and affect the formation of the core values of employees directly, and improve the core competitiveness of enterprises.

Protect Security of Big Data. How to protect the security of big data? Technology is the first layer of security data security, and the good management is the core. The first, we should construct standard systems to promote the order of big data management. The second, we should set up a monitoring system to control the data strictly and prevent information leakage. The third, we need to improve technology of cloud computing to find the new foothold. Strengthen the cloud computing technology innovation capability could provide a better platform for data mining, analysis and other services.

Government Should Legislate. Strengthen the leadership of the government will allow more companies to study and develop large data. The government needs to introduce information security laws and improve the existing big data legislation. Many countries clearly pointed out that enterprises in the mobile phone and the use of personal sensitive information should first get a permission of personal information. In the future, we believe that Society will be balance of technology development and information rights protection and achieve maximum benefit.

\section{References}

[1] GantzJ, ReinselD. "Digital Universe Study: Extracting VaLue from chaos”. IDC. Go-to-Market Services, 2011, pp. 15-16.

[2] J.Liu, "Amazon.cn, to build the next generation of electronic commerce" China Internet Week,March.2010,pp.54-55.

[3] Z.Zhao, X. Wu, "Supply chain management based on internal and external customer demand", Enterprise Reform and Management,2006,pp.8-9,doi:10.13768/j.cnki.cn11-3793/f.2006.03.004.

[4] J.Sun, Amazon: warehouse change lead to change supply chain, Chinese Business News,Nov.2008,(C07).

[5] G. Akerlof, The market for "lemons": Quality uncertainty and the market mechanism, Quarterly Journal of Economics, 1970, pp. 488-500.

[6] Pavlou PA, Understanding and mitigating uncertainty in online exchange relationships: A Principal-agent perspective, MIS Quarterly, 2007, pp. 105-36.

[7] Brad Stone, Amazon: tyrant with a fighter, Business(Review),2013,pp.116-119 
[8] M.Liu, Beyond power: leadership in the Internet era, Beijing, China National School of Administration,2011Z.Wang, The United States to promote the development of the strategic value of big data technology, China Development Observation,2012(6),pp.44-45.

[9] Lynch,R, Strategic Management, Pearson Education Canada;2011.6,pp.7-8.

[10] Z.Wang, The United States to promote the development of the strategic value of big data technology, China Development Observation,2012(6),pp.44-45.

[11] "Enterprise innovation lies in the quality of data management of big data 89\%",Internet Information Research Institute of Communication University of China,Nov.2014. 